\title{
Oral Cancer: After Effects and Improving the Outcome
}

Cancer of the oral and maxillofacial region can lead to potentially severe compromises in the quality of life of the affected individual. A combined planned treatment efforts from oral and maxillofacial surgeons, surgical oncologists, ENT surgeons, speech therapists, psycho therapists and maxillofacial prosthodontists can help the patient lead a normal life with none or minimum post surgical complications.

No cancer is minor to the affected individual. The threat of physical, social and emotional well being, together with functioning can vary dramatically among individuals according to the location and severity of the disease. ${ }^{1}$ Oral and other cancers of head and neck can impair the functions of oral and maxillofacial region and also cause cosmetic deformities. This in turn inflicts a lot of psychological trauma to the individual. Such an effect persists even after the surgical treatment. These effects include fear of public appearances and inability to present oneself in intimate, social and work settings. ${ }^{2,3}$ It is estimated that $20-25 \%$ of the patients experience a major depression during their illness. ${ }^{4}$ In addition to this, the patient can also experience fear of death, stigma and ostracism, abandonment, punishment, anxiety, inferiority and chronicity. ${ }^{5}$

Disease of Oro-facial region greatly affects basic functions like eating, swallowing and speaking. The patient cannot chew properly, his eating becomes untidy and slow and he requires special food preparation as his masticatory efficiency is compromised. Difficulty in swallowing is another problem commonly encountered, especially after the removal of large oral tumors. Other problems like altered bolus transport, drooling of saliva, nasal reflux and aspiration, weight loss and dehydration are also not uncommon. Speech, a combined effort of various organs of the oral, nasal, laryngeal and pharyngeal region, can be greatly impaired and at times even lost if one or more of such organs are surgically removed. Speech serves emotional function such as maintain psychological equilibrium during periods of acute stress. This absence of controlled speech may lead to increased tension and frustration. ${ }^{6}$

An individual's face shows emotion, demonstrates intellect, and is a vehicle for communication. It is also a basis of judgment of attractiveness and is the main source of identity or self esteem. ${ }^{4}$ Surgery of the oral and maxillofacial region especially those done for carcinomas, leaves a severe mark of disfigurement and a person with such disfigured face will be more or less isolate, feel inferior and also will be denied of opportunities. His own face can become a misfortune to him.

Considering the number of problems an oral cancer patient faces even after the treatment, rehabilitation of such individual becomes very crucial. The prime focus should be restoring the functions and ultimately improving the esthetics. Various forms of oral and maxillofacial prostheses can serve as the missing portion of the surgical defect.

For rehabilitation of palatal defects a variety of obturators ranging from simple palatal obturators covering small palatal defects to larger ones extending up to and supporting the nasal cavity, the zygomatic arch, cheek, and even the orbital floor can be fabricated. Such obturators not only improve the appearance of the deformed face but also restore the functions of chewing, swallowing and speaking. These prostheses close the oro-nasal communication, the teeth placed on them help in chewing and the hollow bulbs extending into the nasal cavity help to improve the resonance of voice. 
For mandibulectomy cases where reconstruction with grafts have been done, a mandibular prosthesis can be constructed which can restore the lost portion of the mandible, and the teeth attached to the prosthesis can help in mastication. In cases where grafts have not been used, the remaining mandible usually tends to get displaced leading to disocclusion of the remaining teeth. The mandibular guide flange prosthesis can help the mandible to go back to its normal position.

For improving the speech, for correction of the velopharyngeal function or for restoration of soft palate defects different types of prostheses like special obturators with extensions up to the soft palate and pharynx and palatal lift prosthesis can be fabricated, which not only improve the function but also provide the bulk which masks the defect. Auricular, ocular, orbital, nasal and facial prosthesis can fill up and cover the surgical defects in the oral and maxillofacial region and bring back the confidence that an oral cancer patient desperately needs.

\section{REFERENCES}

1. Gotay C, Moore T. Assessing quality of life in head and neck cancer. Qual Life Res. 1992;1:5.

2. List M, Ritter-Sterr C, Lan ky S. A performance status scale for head and neck cancer patients. Cancer. 1990;66:564.

3. Richardson J, Bourque L. Communication after laryngectomy. J Psychosoc Oncol. 1985;3:83.

4. Depression guidelines panel. Detection and Diagnosis. Clinical practice guideline, Number 5. In: Depression in Primary Care. Rockville: U.S. Department of Health and Human Services, Public health service agency for health care policy and research;1993. Publication No. 93-0550.

5. Beumer J, Curtis TA, Marunick MT. The dental clinician and head and neck cancer patient. In: Ross BR, editor. Maxillofacial Rehabilitation. Prosthodontic and Surgical Consideration. St.Louis. Tokyo: Ishiyaku EuroAmerica Inc.; 1996. p. 15-23.

6. Beumer J, Curtis TA, Marunick MT. Behavioral and psychosocial issues in head and neck cancer. In: Gritz ER, Hoffman A, editors. Maxillofacial Rehabilitation. Prosthodontic and Surgical Consideration. St.Louis. Tokyo: Ishiyaku EuroAmerica Inc.; 1996. p. 1-14. 